\title{
Evaluation of the TRIP13 level in breast cancer and insights into potential molecular pathways
}

Jin Lan

Third Affiliated Hospital of Southern Medical University

Jingzhan Huang

Third Affiliated Hospital of Southern Medical University

\section{Yuan Gao}

Third Affiliated Hospital of Southern Medical University

Jingbo Sun

southern medical university

Longshan Zhang

Nanfang Hospital, Southern Medical University

Yunyao Deng

Third Affiliated Hospital of Southern Medical University

Lixin Liu

Third Affiliated Hospital of Southern Medical University

Xiaolong Liu ( $\square$ Ixl1979@i.smu.edu.cn )

Third Affiliated Hospital of Southern Medical University

\section{Research}

Keywords: TRIP13, breast cancer, metastasis, bioinformatic analysis, prognosis

Posted Date: August 12th, 2020

DOI: https://doi.org/10.21203/rs.3.rs-19358/v2

License: (c) (i) This work is licensed under a Creative Commons Attribution 4.0 International License.

Read Full License

Version of Record: A version of this preprint was published at Journal of Cellular and Molecular Medicine on March 23rd, 2022. See the published version at https://doi.org/10.1111/jcmm.17278. 
The authors have withdrawn this preprint from Research Square 